\title{
Un ethos d'auteur africain ou comment déjouer les stéréotypes : le cas de Mission terminée de Mongo Beti
}

An African Author's Ethos or How to Foil Stereotypes: the Case of Mission to Kala by Mongo Beti

\section{Tal Sela}

\section{OpenEdition Journals}

Édition électronique

URL : http://journals.openedition.org/aad/1676

DOI : $10.4000 /$ aad. 1676

ISSN : 1565-8961

Éditeur

Université de Tel-Aviv

\section{Référence électronique}

Tal Sela, « Un ethos d'auteur africain ou comment déjouer les stéréotypes : le cas de Mission terminée de Mongo Beti », Argumentation et Analyse du Discours [En ligne], 12 | 2014, mis en ligne le 20 avril 2014, consulté le 10 décembre 2020. URL : http://journals.openedition.org/aad/1676 ; DOI : https:// doi.org/10.4000/aad.1676

Ce document a été généré automatiquement le 10 décembre 2020.

\section{(c) $($ ) $\odot \ominus$}

Argumentation \& analyse du discours est mis à disposition selon les termes de la licence Creative Commons Attribution - Pas d'Utilisation Commerciale - Pas de Modification 4.0 International. 


\title{
Un ethos d'auteur africain ou comment déjouer les stéréotypes : le cas de Mission terminée de Mongo Beti
}

\author{
An African Author's Ethos or How to Foil Stereotypes: the Case of Mission to \\ Kala by Mongo Beti
}

Tal Sela

\section{Introduction}

Dans son roman Mission terminée (1957 [1985]), Mongo Beti construit une image d'auteur, ou ethos auctorial, qui s'écarte sensiblement de celle qui avait été popularisée par les écrivains et artistes noirs regroupés à Paris autour du mouvement littéraire de la Négritude. Cet ethos préalable (Amossy 2010) d' « écrivain noir/africain » se rapporte à des représentations préexistantes qui circulent dans le discours social, et qui jouent un rôle important pour le déchiffrement de l'œuvre et pour le positionnement de son auteur dans le champ littéraire français. Élaborer une littérature originale nécessite en effet, selon Bernard Mouralis, de prendre en compte «tous ces autres textes produits en Afrique ou à propos de l'Afrique et qui forment ce réseau au centre duquel se trouve placé l'écrivain africain » (1981: 186). Mouralis privilégie la dimension dialogique et interactionnelle qui structure le discours littéraire (comme tout discours). Ce principe suppose à la fois un recours aux stéréotypes dont se nourrit le discours littéraire africain émergent, et un incessant travail de reformulation et de transformation (Amossy 2009). C'est ce travail qui nous intéresse ici : il s'agit de voir comment il est mis en œuvre par les écrivains qui, dans les années 1950, ne voulaient plus refléter l'image convenue d'auteur en "Africain authentique" à laquelle étaient accoutumés les lecteurs français de l'époque. 
2 Rappelons que dans le numéro 3 de la revue Argumentation et Analyse du Discours, une confrontation systématique de la notion d'ethos au problème de l'écriture fictionnelle a permis de « repenser l'instance auctoriale, de redéfinir ses fonctions et d'en éclairer les enjeux » (Kahan 2009: 10). Elle se greffe sur la question plus générale posée sur les apports théoriques de l'AD dans les études littéraires (Amossy et Maingueneau 2004). Plus récemment, le dernier numéro de Contexte (2013) a réexaminé le concept d'ethos à l'intention des jeunes chercheurs en littérature qui portent ces dernières années un intérêt accru aux concepts développés par l'apparat méthodologique de l'AD (Reindert, Dhondt, Horemans, Vanacker et Vandemeulebroucke 2013). La revue entend préciser les contours flous de certains concepts comme celui de posture (Jérôme Meizoz 2007, 2011, 2011b, 2012), d'image (José-Luis Diaz 2000, 2007, 2012) ou encore d'ethos d'auteur (Maingueneau 1993, 2004, 2012, Amossy 2004, 2009, 2010)1.C'est à la lumière de ces travaux qu'on analysera l'interaction entre diverses instances narratives pour y examiner l'ethos, ou présentation de soi, que chaque locuteur construit dans son discours, et la façon dont ces diverses images de soi se recoupent ou se superposent dans le texte. La question qui se trouve au centre de cette étude est de comprendre d'abord comment les ethè discursifs que produisent tous ces intervenants (narrateur, protagoniste et personnages) sont constitutifs de l'ethos de l'auteur dont le nom figure sur la couverture du roman; ensuite, de voir comment cette construction intratextuelle s'articule avec l'image de l'« écrivain africain » qui circule à l'extérieur de l'œuvre fictionnelle.

3 Mon analyse du discours littéraire prendra donc en compte la complexité de l'ethos d'auteur qui résulte de la polyphonie des instances d'énonciation dans la narration. Pour étudier concrètement le récit, j'emprunte à la narratologie certains principes et instruments conceptuels que je croise avec la notion d'ethos discursif, empruntée à l'AD et à l'argumentation dans le discours. Je montrerai comment un ethos auctorial se construit à travers les différents niveaux d'interaction sur lesquels se déploie le récit. À chaque niveau d'interaction se révèle une voix, un JE parlant. L'auteur est celui qui gère les différents niveaux d'interactions ainsi que les différents JE qui prennent successivement en charge l'allocution. L'auteur nous parle ainsi au travers des thèmes choisis, de la mise en intrigue, des images utilisées, de son style. Il nous entretient de sa personne même lorsqu'il n'en traite pas directement et qu'il se dissimule derrière son texte (Amossy 2009, 2002). Je soutiendrai que l'ethos auctorial de Mongo Beti contribue à la force d'un discours qui entend agir sur le lecteur pour infléchir, voire modifier, ses représentations à l'égard de l'« écrivain africain/noir ».

4 Je m'attacherai plus particulièrement à la manière dont les locuteurs ou, en l'occurrence, les instances narratives, construisent leur identité dans ce que Dominique Maingueneau appelle une scène d'énonciation - ou "scénographie » - qui définit les statuts d'énonciateurs et de co-énonciateurs, ainsi que l'espace (topographie) et le temps (chronographie) dans lesquels se développe l'énonciation (Maingueneau 2004, 1993). Étudier la scénographie c'est, dans le cas présent, s'interroger sur la construction de l'identité du sujet parlant à travers la façon dont il se positionne vis-àvis de l'espace (africain) dans lequel il produit son discours. Nous allons donc relier la question de savoir qui parle à celle de savoir comment le même locuteur perçoit l'espace dans lequel il est situé.

5 La première partie de cet article problématise la notion d'« écrivain noir/africain ». La seconde se penche sur le paratexte du roman, à savoir la présentation de l'auteur par 
son éditeur dans la quatrième de couverture, encore imprégnée de l'ethos collectif de l'« écrivain africain ». Enfin, une analyse serrée du discours romanesque doit permettre de préciser le nouvel ethos que travaille à construire l'auteur.

\section{Mongo Beti et le problème de l'« écrivain africain »}

6 Les notions de « littérature africaine » et d'« écrivain africain » ne vont pas de soi et ont fait l'objet d'une vive polémique. Il existe à cet égard deux approches critiques. Il y a d'abord celle de la collection « Continents Noirs » parue en 2000 aux Éditions Gallimard. Son directeur, l'écrivain et traducteur Jean-Noël Schifano, disait qu'elle serait "consacrée aux écritures africaines, principalement d'expression française, en réunissant des textes littéraires ou des essais, contemporains, dus à des écrivains du continent noir et, plus souvent, de sa diaspora ${ }^{2}$. L'expression " écritures africaines " suppose une identité africaine des auteurs ainsi que des thématiques dans lesquelles ils s'investissent. Cette identité de l'auteur déterminerait l'«africanité » de son écriture comme forme et contenu spécifique.

7 A l'initiative de Gallimard de réunir des écrivains qui s'affirment comme « africains » et produisent - résultat obligé - une « littérature africaine », s'opposent les signataires du manifeste Pour une littérature-monde en français paru en 2007 également chez Gallimard. Ceux-ci contestent la dénomination « littérature africaine » qu'ils considèrent comme une catégorie de la francophonie littéraire, concept que l'on applique, selon Alain Mabankou, à des écrivains non français pour instaurer une séparation, voire une hiérarchie par rapport à la "littérature française ». Ils s'insèrent en fait dans un mouvement plus large d'écrivains contemporains qui récusaient depuis déjà quelques années l'étiquette de "littérature africaine ». Abdouraham Waberi, un autre signataire du manifeste, fait référence à Kossi Efoui décrétant dans une interview de 2004 que « la littérature africaine n'existe pas ». Efoui développe un argument, largement repris depuis, selon lequel « quand Sony Labou Tansi écrit, c'est Sony Labou Tansi qui écrit, ce n'est ni le Congo, ni l'Afrique $»^{3}$. Selon lui, le discours littéraire émane d'un auteur et non d'une collectivité, et est l'expression d'une originalité artistique plutôt que d'une identité culturelle, qu'elle soit africaine ou autre.

8 La polémique suppose donc deux attitudes critiques: la première, à l'exemple de "Continents noirs », envisage la fiction à l'aune d'une certaine réalité historique et politique à laquelle se réfère le roman et dont l'auteur est issu. Cette tradition critique n'est pas nouvelle. Déjà en 1974, Mohamadou Kane notait que le romancier africain « reste toute sa vie marqué de façon indélébile par cet environnement socio-culturel. Quel que puisse être, par la suite, son degré d'adaptation à la culture française, il sera toujours impossible d'expliquer son œuvre en faisant abstraction de son origine » (1974: 549). La critique d'Eloïse Brière s'inspire de cette approche quand elle reproche à Dorothy Blaire de relever que les intertitres des chapitres de Mission terminée de Mongo Beti, formulés en guise de résumé, font écho à des procédés employés dans le roman picaresque du $18^{\mathrm{e}}$ siècle, au lieu de « faire appel à la littérature orale traditionnelle qui comporte bien des exemples de ce genre et qui est certainement plus près du contexte littéraire dont est sorti Mongo Beti » (Brière 1979 : 78). Pour soutenir cette assertion, Brière se réclame de Mongo Beti lui-même qui assure que de tels résumés sont fréquents dans la littérature orale, le Mvett en particulier. 
Pour elle, cette note confirme l'inclination naturelle de l'écrivain envers les littératures traditionnelles de son pays, et suffit pour réfuter la thèse de Dorothy Blaire.

L'autre attitude, représentée par le Manifeste pour une littérature monde, s'insère elle aussi dans une longue tradition critique. Elle privilégie, à l'exemple de Dorothy Blaire, une perspective comparative qui veut rattacher l'œuvre de Mongo Beti non pas au contexte culturel africain mais à la "république mondiale des lettres". Bernard Mouralis, qui a étudié les influences littéraires qui se sont exercées sur Mongo Beti, rappelle la forte impression qu'ont produite sur lui, lorsqu'il était lycéen, les textes de la littérature française. Mouralis se réclame lui aussi de Mongo Beti pour prouver le lien aux traditions littéraires françaises. Voici comment Beti explique l'exploitation, dans son œuvre, de la figure rhétorique de l'ironie :

En ce qui concerne l'ironie mordante, comme je vous l'expliquais ce matin à propos de Candide et de Lettres persanes, puisque je suis de culture française, j'ai été forcément marqué par des ouvrages comme ceux-là. [...] Comme le montre Voltaire, que je considère comme le grand maître de l'ironie, je pense que le lecteur est très sensible à l'ironie et surtout celui qui en est victime montre très vite que l'ironie lui fait mal (Beti, Mouralis $1981: 25$ ).

10 Les écrivains africains eux-mêmes ont pris une part active dans cette remise en cause de leur statut. Déjà vers 1950, certains doublent leurs textes de fiction d'un discours critique destiné à définir la littérature africaine et son auteur (Mouralis 1981). En témoignent le $\mathrm{I}^{\mathrm{er}}$ et le $\mathrm{II}^{\mathrm{e}}$ Congrès international des Ecrivains et Artistes noirs à Paris en 1956 et à Rome en 1959. Sous l'égide des chantres de la Négritude, des sortes de «loiscadres » y sont élaborées, dictant les règles du discours littéraire africain en langue française. Elles s'articulent autour de deux principes ainsi résumés par Mouralis :

Il faut d'abord que l'écrivain se réfère, à la fois pour la transmettre et l'illustrer, à une authentique culture négro-africaine ; il faut ensuite que son travail d'écrivain aille dans le même sens que les luttes menées parallèlement sur le plan politique et social et que son œuvre ne soit rien d'autre en définitive que l'expression de ces luttes (1981 : 465).

11 La critique des écrivains joue ainsi un rôle éminent dans l'institutionnalisation de leurs discours littéraires. Elle implique un certain ordre du discours qui donne de l'Afrique l'image d'un espace culturel unifié fondé sur une unité artistique.

Cependant, la polémique autour du sens à donner à la notion d'« écrivain africain » s'est déclenchée dès les années 50-60 où des romanciers africains se sont mis à contester les normes prescrites par les pères fondateurs de la Négritude. Ils cherchent dès cette époque à s'affirmer non pas comme «écrivains africains " mais comme «écrivains» tout court (Kesteloot 1963, Killam 1975). Le Camerounais Mongo Beti a donné l'exemple en 1955 en publiant dans la revue Présence africaine un article intitulé Afrique noire, littérature rose.

13 L'article est important à nos yeux pour sa dimension de manifeste et de programme, qu'il convient d'abord de contextualiser dans l'entreprise générale de ce numéro de Présence Africaine. Dans son article, "The Cultural Politics of the Early Présence Africaine, 1947-55 (1999), Hassan D. Sallah relie cette entreprise aux circonstances historiques de la Conférence de Bandung, à la création consécutive du bloc des pays non-alignés, du début de la Guerre d'Algérie ainsi qu'à l'impact de la guerre froide sur les anciens empires coloniaux. Ces événements donnent un fort élan au mouvement intellectuel de lutte pour l'Indépendance des pays africains qui se concrétise dans l'action de Présence africaine. Sallah soutient que l'entreprise de Présence africaine 1955 est radicalement 
opposée au projet culturel que la revue avait voulu lancer en 1947. Son article procède à une comparaison entre le numéro inaugural de 1947 et celui de 1955. Il montre que les événements politiques entre les deux éditions ont été si dramatiques que le Comité de Présence africaine 1955 a jugé nécessaire de prendre un nouveau départ et de le souligner par la réinitialisation de la numérotation de la revue. Sallah relève les changements explicites dans la structure éditoriale concernant la décision de se dispenser du Comité de Patronage, constitué par des intellectuels blancs. La revue a aussi renoncé à présenter les contributeurs selon une hiérarchie tenant compte de l'appartenance raciale de l'auteur (Blancs en tête) et de leur réputation dans les milieux intellectuels. Ces modifications éditoriales se conforment aux objectifs de la revue qui rejette la politique d'assimilation des Africains prônée par la Quatrième République et se veut solidaire de la lutte antiraciste et anticoloniale. L'auteur signale enfin la réorientation thématique de la revue d'inspiration marxiste et nationaliste. Dans le domaine artistique, Sallah constate la tendance à remplacer l'image littéraire de l'Afrique, idyllique, harmonieuse et naturelle, telle qu'elle a été dessinée par les écrivains de la Négritude ${ }^{4}$, par un portrait réaliste de la situation coloniale, aussi violente et repoussante soit-elle.

Cette courte contextualisation, à la fois historique et éditoriale, élargit notre lecture de l'article de Mongo Beti Afrique noire, littérature rose, et permet de mesurer toute la portée de son argumentation. Beti constate qu'il n'existe pas de littérature de langue française de qualité inspirée par l'Afrique. Il évoque, dans cette perspective, L'enfant noir de Camara Laye pour reprocher aux écrivains africains de donner dans le pittoresque, voire dans le fantastique, ce qui, selon lui, leur fait écrire des « sottises ». Il est partisan d'une écriture réaliste qui seule, selon lui, permet de porter un regard juste sur le fait colonial. Il s'oppose par-là à l'idée que l'écrivain africain possède une originalité qu'il tire de son africanité, et soutient que la qualité de l'œuvre littéraire ne dépend pas de l'«origine ethnique» de l'auteur, ni de sa "race», mais de sa "personnalité » ainsi que de sa "sensibilité à l'attitude du public ». Ainsi, Mongo Béti présente entre les lignes un programme artistique à travers lequel il cherche à se positionner comme écrivain singulier, différent des autres. L'ethos d'écrivain transgresseur et novateur, anti-racial, antiraciste et anticolonialiste qu'il construit, répond à l'entreprise générale de Présence africaine.

Il convient à présent de se demander quelles suites concrètes a connu le programme artistique que Mongo Beti élabore en théorie dans Afrique noire, littérature rose et si l' ethos préalable d'« écrivain africain " s'accorde en pratique avec celui qui se construit ultérieurement dans son œuvre littéraire. À cet effet, nous étudierons le troisième roman de Mongo Beti, Mission terminée.

Bien que le commentaire éditorial rapproche l'œuvre du genre du roman d'aventure («Et voici notre adolescent parti pour des aventures qui vont bouleverser son destin »), nous verrons que la mission que le protagoniste doit accomplir prend la forme d'une quête d'identité culturelle qu'il mènera à travers les lieux communs et les stéréotypes associés à l'Afrique et aux Africains. Dans cette perspective, la question de savoir comment la construction intratextuelle de l'identité du narrateur-protagoniste s'articule avec l'identité auctoriale nous semble particulièrement intéressante ${ }^{5}$. Étudier les liens qui se tissent entre la quête identitaire du héros avec celle, dissimulée, de l'auteur, sans pour autant rabattre la figure de l'un sur celle de l'autre - telle est la tâche que s'assigne l'analyse qui suit. 


\section{Analyse discursive de l'ethos du narrateur}

Pour présenter les niveaux sur lesquels va se construire un ethos d'auteur, je distingue les interactions qui se déroulent sur le niveau paratextuel (épitexte, épigraphe, épilogue, intertitres, prologue... ${ }^{\text {}}$ ) de celles qui se forment à l'intérieur du texte narratif qui raconte, par le biais d'une instance de narration, une histoire constituée d'une série d'événements concernant des personnages ${ }^{7}$. J'étudie d'abord le niveau paratextuel et, notamment, les informations que nous offrent la quatrième de couverture. Le commentaire éditorial de celle-ci donne l'impression d'une cohérence entre l'ethos préalable (de type collectif) de l'Africain - conformes aux stéréotypes - et l'ethos (éditorial) de l'auteur. Pourtant la photo de l'auteur, qui s'affiche sur le dos du livre, trahit une certaine tension.

\subsection{Analyse paratextuelle}

Le fait de prendre un livre entre les mains signale l'entrée du lecteur dans le jeu interactionnel avec le texte qu'il lit. En effet, le titre du livre, le nom de son auteur, les photos qui les accompagnent constituent une interaction préliminaire. Les informations que donne le péritexte éditorial de Mission terminée méritent qu'on s'y arrête.

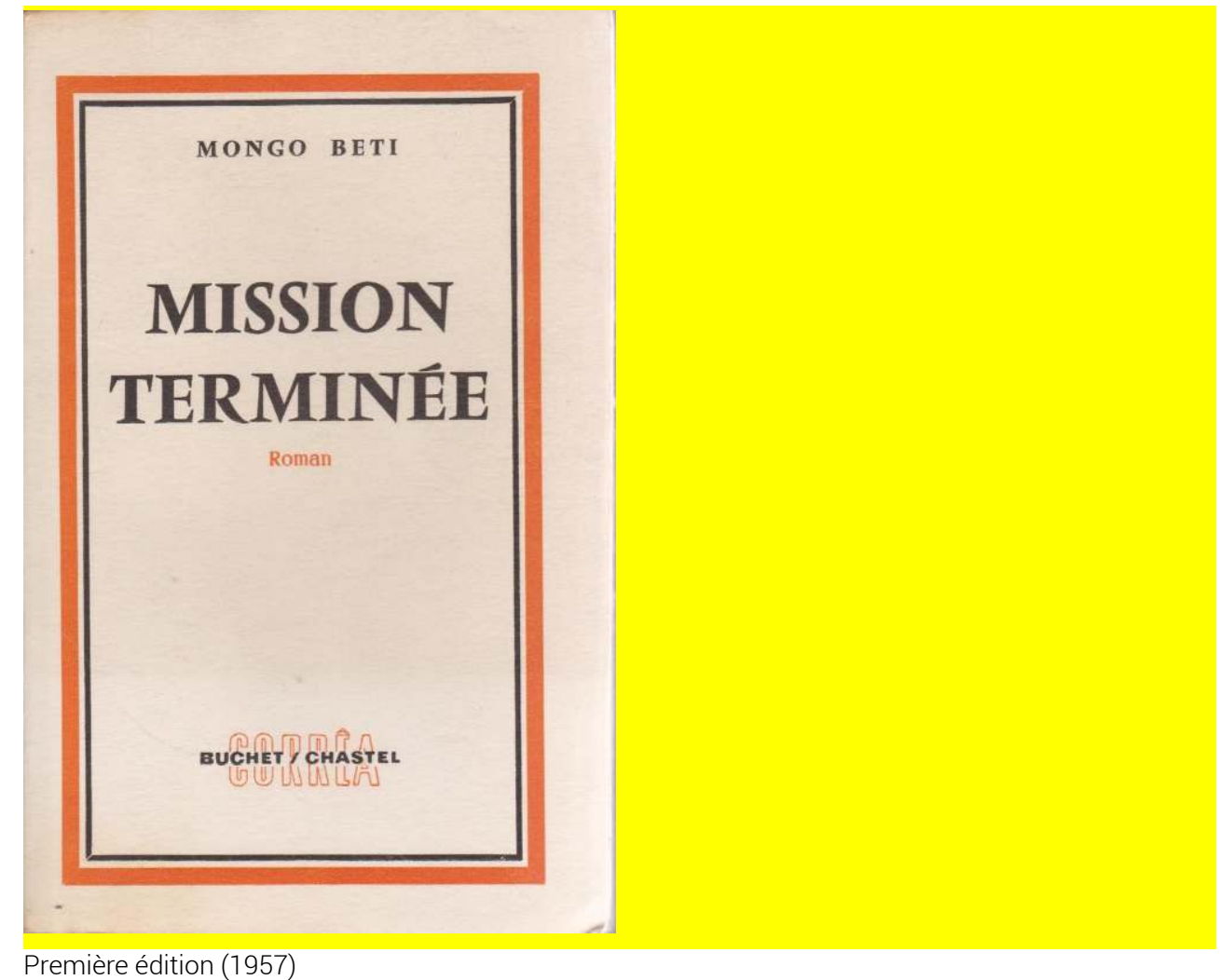




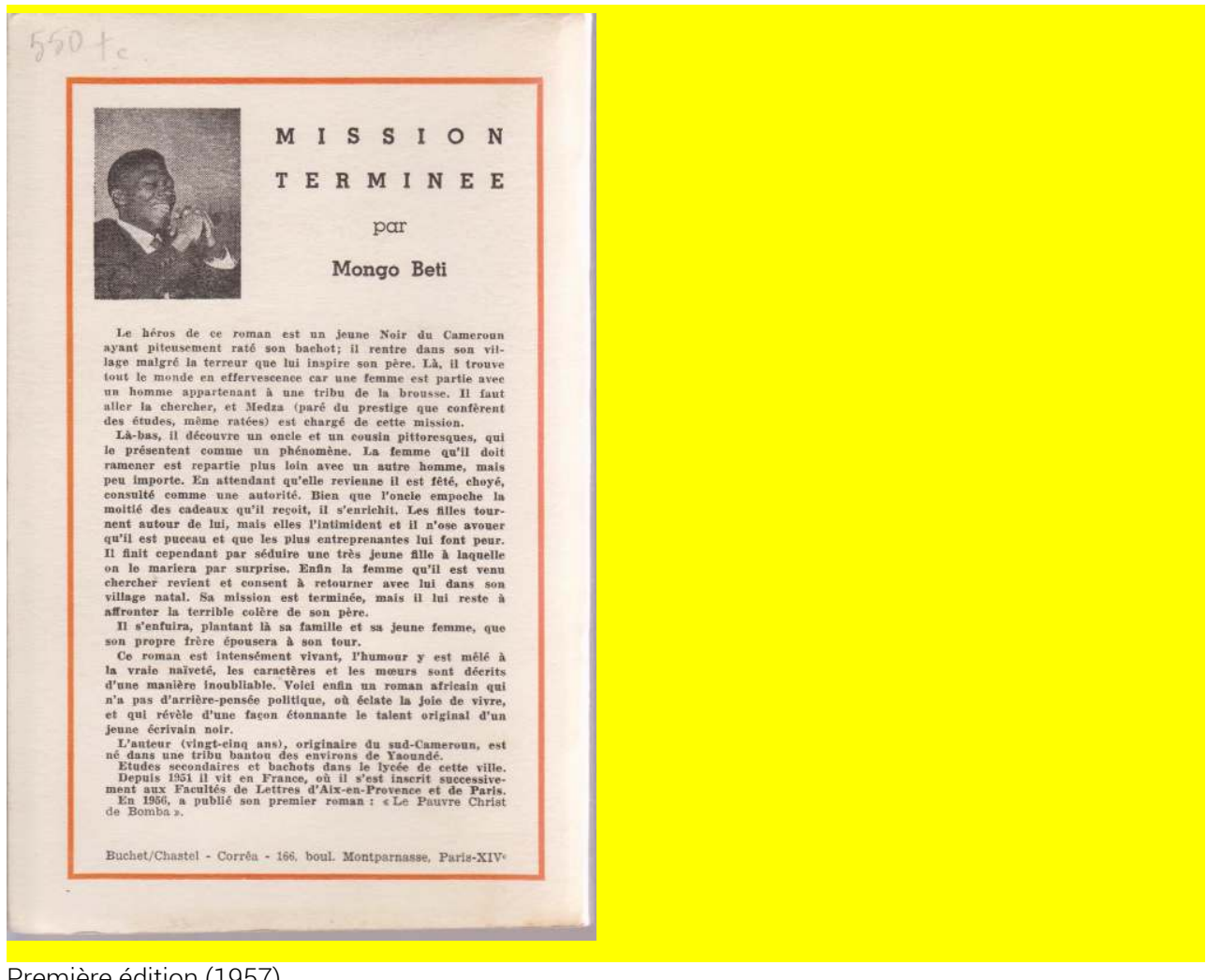

Première édition (1957)

Le commentaire de la quatrième de couverture émane en principe de l'éditeur (même s'il est en général soumis à l'auteur avant la publication). Il comprend trois parties et sa version de 1957 est plus longue que celle de la deuxième édition, publiée en 1985 en livre de poche ${ }^{8}$.Le début est un résumé du roman. Il est suivi par un jugement de valeur très positif sur le roman et le romancier ${ }^{9}$ :

Ce roman est intensément vivant, l'humour y est mêlé à la vraie naïveté, les caractères et les mœurs sont décrits d'une manière inoubliable. Voici enfin un roman africain qui n'a pas d'arrière-pensée politique, où éclate la joie de vivre, et qui révéla d'une façon étonnante le talent original d'un jeune écrivain noir.

L'éditeur commence par un constat: Mission terminée est un roman "intensément vivant ». Il lie ensuite l'« humour » à la "naïveté » des personnages pour insister sur le talent dont fait preuve l'auteur pour décrire "les caractères et les mœurs" des Africains. On remarque la connotation, aux relents coloniaux, de l'expression « la vraie naïveté » avec la manière de faire jouer la fibre ethnologique du lecteur en évoquant «les caractères et les mœurs» des Africains. L'exonération de toute pesanteur politique ("Voici enfin un roman africain qui n'a pas d'arrière-pensée politique ») dégage une impression de légèreté, voire d'euphorie, qui fait écho à la volonté présumée de l'auteur de donner libre cours à la «joie de vivre» africaine. La photo voisine de Mongo Beti tout sourire, redouble le message. L'éditeur distingue ainsi, d'un côté, l'auteur, qui esquisse un portrait d'une société africaine, et de l'autre, son écriture: vivante, humoristique et joviale. Mais la proximité des deux jugements suggère un rapport étroit entre les deux : les locutions « intensément vivant », «joie de vivre ", « humour », " naïveté », permettent à l'éditeur de transposer les qualités qu'il attribue au roman à celles de la culture africaine qu'il décrit. La figure de l'homme noir qui est ainsi suggérée ressemble fort à l'image d'Épinal de l'Africain : il est spontané, sans artifice, d'une gaieté naïve, franche et naturelle. On retrouve tout le registre des 
stéréotypes traditionnels auxquels est accoutumé le lecteur français, résumés par la notion de «bon sauvage ».

La troisième partie du commentaire éditorial est une notice biographique :

L'auteur (vingt-cinq ans), originaire du sud-Cameroun, est né dans une tribu bantou des environs de Yaoundé.

Etudes secondaires et bachots dans le lycée de cette ville. Depuis 1951 il vit en France, où il s'est inscrit successivement aux Facultés de Lettres d'Aix-en-Provence et de Paris. En 1956, a publié son premier roman : «Le Pauvre Christ de Bomba.»

Cette biographie pointe deux composantes identitaires de l'auteur. L'une, innée et collective, provient de son appartenance tribale ; l'autre, acquise et individuelle, se réfère à sa formation scolaire et universitaire, au terme de laquelle il parvient au sommet de la hiérarchie culturelle en devenant romancier. L'image éditoriale de l'auteur se construit ainsi par l'intermédiaire d'un ethos préalable figé d'homme noir, comme le prouve la catégorie d'« écrivains noirs » dans laquelle on le range d'emblée ${ }^{10}$. On fait ainsi endosser à l'auteur tous les stéréotypes que l'éditeur attribue à l'Africain.

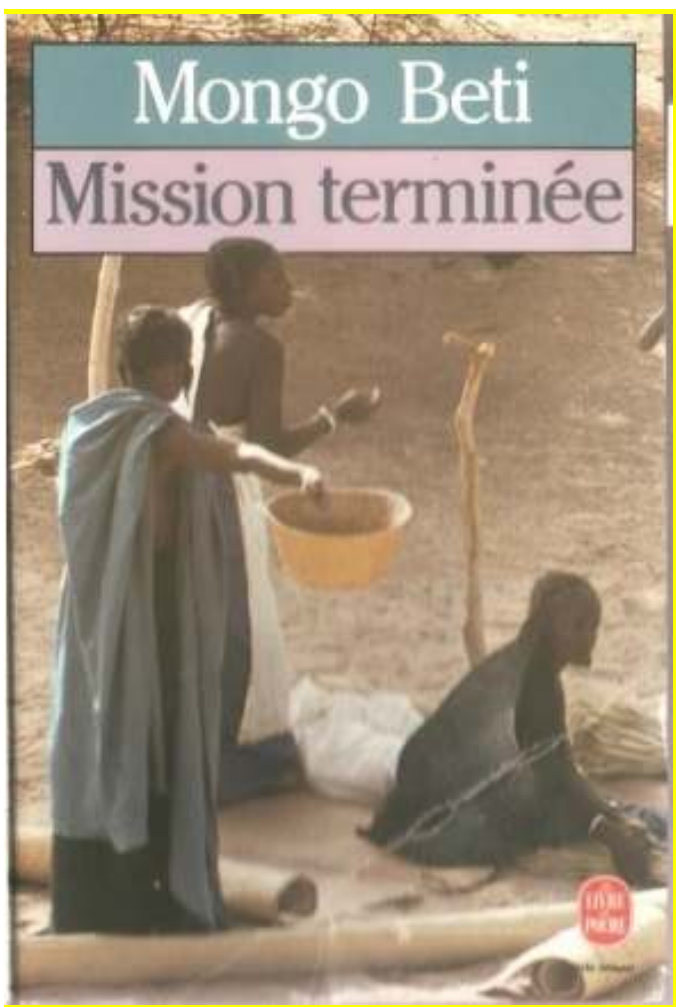

Deuxième édition 1985 


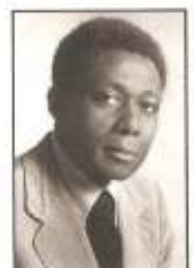

Le héros de ce roman est un jeune Noir du Cameroun. Ayant piteusement raté son bachoot, it rentre dans son village malgré lì terreur que lui inspire son pére. effervescence car une femme est partie avec un homme apparte nant à une tribu de la brousse. II faut aller la chercher. Mezda (paré du prestige que confèrent des études, mème ratées) est chargé de cette mission

Et voici notre adolescent parti pour des aventures qui vont bouleverser son destin.

Mission terminée de Mongo Beti, un des meilleurs cerivains noirs contemporains, est un roman inten sément vivant où édate la joie de vivre : l'humour y est mélé a la vraie näveté et les caractéres, les mours, sont décrits d'une manière inoubliable.

Prix Sainte-Beuve roman,

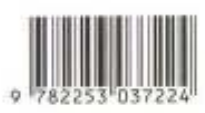

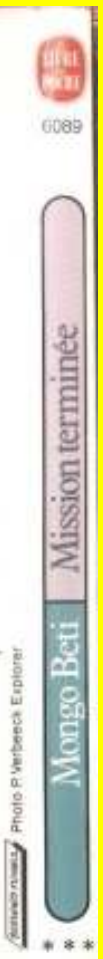

Deuxième édition 1985 Mongo Beti de 1957 a changé de pose. On le voit toujours en costume-cravate mais avec un visage inexpressif cette fois-ci comme sur les photos d'identité. Il offre l'image classique d'un écrivain-intellectuel qui tranche radicalement avec le stéréotype du « bon sauvage » que véhicule le commentaire en vis à vis. Qu'il soit frivole ou sérieux, la tenue costume-cravate à l'occidentale est aux antipodes de la figure traditionnelle de l'homme noir, elle représente même ce qu'il y a de plus inauthentique dans l'existence nègre exaltée dans le discours littéraire porté par les poètes de la Négritude. C'est ce qu'écrit, par exemple, Aimé Césaire dans la revue L'Étudiant noir, publiée à Paris en mars 1935 :

Un jour, le Nègre s'empara de la cravate du Blanc, se saisit d'un chapeau melon, s'en affubla, et partit en riant...

Ce n'était qu'un jeu, mais le Nègre se laissa prendre au jeu : il s'habitua si bien à la cravate et au chapeau melon qu'il finit par croire qu'il les avait toujours portés; il se moqua de ceux qui n'en portaient point et renia son père qui a nom Esprit de Brousse...C'est un peu l'histoire du Nègre d'avant-guerre qui n'est que le Nègre d'avant-raison. Il s'est mis à l'école des Blancs : il a voulu devenir «Autre »: il a voulu être « assimilé ».

Un autre exemple est celui de Léon Gontran Damas, considéré, avec Césaire et Senghor, comme co-fondateur du mouvement de la Négritude. Damas introduit dans Pigment, un recueil de poèmes qu'il publie en 1937, avec une gravure sur bois de Frans Masereel : 


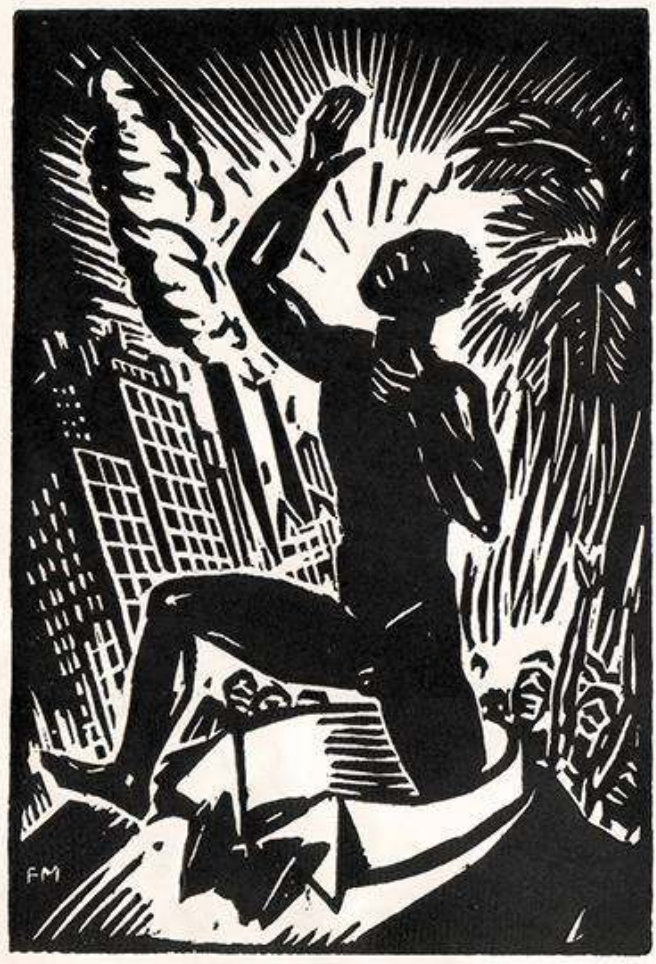

Frans Masereel, gravure sur bois (1937) authentique. Doit-on s'attendre à ce que l'Africain à Paris reconnaisse son originalité culturelle, se délivre de sa tenue de soirée et communie avec la nature ? Qu'est-ce qui détermine en l'occurrence l'identité de l'auteur? Est-ce plutôt son statut d'écrivainintellectuel, ou inversement, ses origines africaines? Mongo Beti est-il un sauvage dans la peau d'un intellectuel qui développe, à l'image d'un Dr. Jekyll et Mr. Hyde, une double et contradictoire personnalité ?

Cette gravure, depuis devenue célèbre, montre un homme noir, tiraillé entre la ville et les tropiques, qui s'échappe vigoureusement par le col cassé fermé par un nœud papillon d'une tenue de soirée. Dans un élan triomphal des bras et de la voix, il fuit la ville, acclamé dans son dos par une foule dominée par des palmiers, symbolisant le peuple africain.

La double présentation de l'auteur qui émane du commentaire éditorial et de la photo est révélatrice du caractère intrinsèquement ambivalent de la dénomination « écrivain africain ». Elle comporte deux composantes de sens contraire : d'un côté, l'« écrivain » se rapporte à la classe des intellectuels de type occidental, et de l'autre, l'adjectif " africain » renvoie à la vie libre dans la nature. Ce que le commentaire éditorial fait dire au monde africain - traditionnel, naturel et authentique dont est prétendument issu l'auteur - entre en conflit avec le statut d'écrivain et d'intellectuel qu'il occupe dans le champ littéraire français. Il s'avère que ce qui lie l'Africain à la nature est ce qui lie le roman à un lieu (l'Afrique authentique) dont l'auteur tire sa force mais auquel il s'est arraché. Réfléchir, dans cette perspective, sur l'émergence de l'œuvre de Mongo Beti, c'est considérer la construction contradictoire de l'identité énonciative qui est à la 
fois "prise de position » mais aussi découpage d'un certain imaginaire que l'auteur s'efforce de renouveler.

Confrontons à présent l'ethos éditorial de l'auteur avec celui qui ressort du texte narratif. Mongo Beti parvient-il à faire éclater les stéréotypes sur l'Afrique et l'Africain et à tenir la promesse faite dans Afrique noire, littérature rose d'inventer un nouvel idéal, ou, pour parler en termes d'AD, un nouvel ethos d'auteur africain?

\subsection{Analyse textuelle}

\subsubsection{La dimension autobiographique du récit}

Parmi les interactions qui se jouent à l'intérieur du texte narratif, on relève l'identité de deux JE : le JE-protagoniste de Medza enfant, qui vit les événements tels qu'ils se produisent ici et maintenant et le JE-narrateur de Medza adulte, qui présente l'histoire sous forme d'autobiographie fictionnelle en se projetant à partir de son propre présent dans son passé d'adolescent. On constate cette dimension autobiographique dans le tout premier énoncé du prologue : "pourquoi est-ce de cette histoire que le souvenir reflue vers moi comme une marée, sans répit, ...", ainsi que dans l'incipit de la première partie: "Chaque fois que je me rappelle ma petite aventure, $j$ 'approuve soudain la vilaine envie de redevenir jeune, de la recommencer ». La relation de cette aventure par le JE-narrateur apparait de fait comme une manière de recommencer l'aventure avec, à son côté, le narrataire comme compagnon de route.

30 Le récit va donc se déployer sous le regard rétrospectif du JE-narrateur. L'image du JEprotagoniste, Medza enfant, se construit dans les interactions constitutives des différentes étapes du périple. Ces interactions se manifestent, d'un côté, par un discours direct: le JE-narrateur rapporte, de mémoire, les dialogues échangés, plusieurs années auparavant, entre le JE-protagoniste et les autres personnages. Ces dialogues apparaissent dans le texte entre guillemets, sous forme de citations. Mais l'image du JE-protagoniste est également construite dans un autre registre de discours élaboré par un JE-narrateur, qui accompagne les interactions de commentaires et d'interprétations. L'image qu'il donne du JE-protagoniste relève d'un discours de type descriptif et explicatif effectué du point de vue du présent de la narration.

31 La construction de l'ethos discursif du JE-protagoniste ainsi que de celui du JEnarrateur participent d'une démarche argumentative dont il est impossible d'évaluer la nature et le fonctionnement sans prendre en considération la dimension d'autobiographie fictionnelle dans laquelle l'argumentation se déploie. Le prologue du roman nous offre quelques indices révélateurs. Le JE-narrateur se demande ainsi :

Pourquoi est-ce de cette histoire que le souvenir reflue vers moi comme une marée, sans répit, aux jours de cafard autant qu'à ceux d'exaltation? [...]

Je n'y peux rien. Tandis que tous mes souvenirs se désagrègent, se liquéfient et finalement s'estompent au soleil de mon âge adulte [...] c'est cette aventure d'adolescent, elle seule, qui tient tête à la déroute avec l'obstination des héros, remplit les vides laissés par la désertion de mon passé, m'envahit, m'imprègne.

Pourquoi?

A-t-elle une signification que je n'ai pas réussi à déchiffrer? Je n'en sais rien. (Prologue) $)^{11}$

32 À travers les questions qu'il se pose, le narrateur fait comprendre que son histoire a pour objet sa propre vie d'adolescent, puis présente les genres qui participent au récit de ses souvenirs, à savoir le récit d'enfance et le récit d'aventure. Il précise aussi 
comment ses souvenirs lui remontent à l'esprit et analyse les émotions qu'ils suscitent, poussés par un élan d'" enthousiasme romantique ", imprégnés de regret mélancolique pour une adolescence sans éclat. Il insiste enfin sur le caractère obsédant des souvenirs dont il ne parvient pas à se libérer et se demande pourquoi ils s'imposent ainsi à l'esprit de façon répétée et incoercible. Ce dernier point est important parce qu'il donne l'impression que la relation de son aventure d'enfance est destinée à épurer, comme dans une thérapie psychologique, un jugement passionnel, voire pathologique, du narrateur sur lui-même. Le narrataire, et à travers lui le lecteur, sont ainsi sollicités par le narrateur pour tirer de son récit la signification que lui-même n'a pas réussi jusquelà à dégager.

33 Il en ressort que si l'ethos du JE-protagoniste - Medza adolescent - se construit par le discours du JE-narrateur - Medza adulte - l'ethos de Medza adolescent est en fait constitutif de l'ethos de Medza adulte. Ensemble ils participent d'une démarche argumentative particulière : faire entrer le lecteur dans un jeu littéraire qui consiste à attribuer une signification à la vie du narrateur, la signification même qui lui a échappée. La distanciation que cette démarche implique entre Medza adulte et Medza adolescent suggère en filigrane l'aspect illusoire d'un réel que le narrateur restitue de mémoire. Ce procédé littéraire insiste en fait non pas sur le réel mais sur l'idée que le narrateur s'en fait. Qui plus est, il présente la construction de l'identité comme un artefact de discours que le narrateur mobilise dans son argumentation. Il est intéressant, dans cette perspective, que l'image (spéculaire) que le narrateur donne du protagoniste s'organise, à travers toutes les étapes de son périple, autour de l'axe thématique de l'exclusion sociale et de l'altérité culturelle. La question qui se pose est de savoir comment cet axe participe de la construction du sens de la vie du narrateur, eu égard à sa manière particulière de construire son identité.

\subsubsection{Analyse du récit}

34 L'événement déclencheur du récit est l'échec scolaire de Medza qui a été recalé à l'oral de son baccalauréat. Le narrateur insiste sur l'intention qu'il avait de rentrer dans son village pour y préparer la deuxième session trois mois plus tard. Il rentre mais son projet de préparer l'examen suivant ne se réalise pas. À peine arrivé, on lui demande de repartir chercher la mariée disparue de Niam dans un village de la brousse (Kala). Medza refuse. Il entre dans une négociation avec Niam, soutenu par Bikoloko le patriarche, et avec, comme public, les autres habitants du village. Pour convaincre son auditoire, Medza s'appuie sur son identité, son ethos de lycéen. Il compte sur l'aura qui entoure l'école coloniale pour faire admettre l'obligation qui est la sienne de réussir son examen, et par là, la nécessité de rester au village. Mais Niam reproche à Medza son esprit individualiste. Aller chercher sa promise sera pour Mezda assumer ses responsabilités de membre de la communauté. Un de ses oncles se réclame des antiques traditions du village pour mettre en cause son neveu devant l'auditoire :

Ce garçon n'a peut-être rien compris à rien ; il n'a certainement rien compris. [...] Pourquoi voulez-vous qu'il comprenne forcément? Je vous le demande. Songez donc, depuis qu'il ne vient parmi nous que par intermittence, depuis qu'il est à l'école, le miracle, ce serait bien plutôt qu'il soit encore à l'aise dans notre sagesse et dans nos coutumes. Au lieu de vous étonner, expliquez-lui donc (27).

35 L'oncle flatte l'auditoire par le rappel des coutumes et de la sagesse que son neveu a perdues. La formation scolaire de Mezda est ainsi présentée tantôt sous un jour 
défavorable : elle a séparé Medza de sa communauté, la mission est le passage obligé pour renouer avec elle; tantôt sous un jour favorable comme le moyen pour Medza d'assurer la réussite de sa mission. C'est ce que lui explique justement le patriarche :

Mais tu es un homme terrible! Et tu parles aussi avec la voix du tonnerre. Et tu ne soupçonnes même pas ta puissance! Ta voix du tonnerre, sais-tu ce que c'est? Tes diplômes, ton instruction, ta connaissance des choses des Blancs. Sais-tu ce que s'imaginent sérieusement ces bushmen de l'arrière-pays? Qu'il te suffirait d'adresser une lettre écrite en français, de parler en français au chef de la subdivision la plus proche, pour faire mettre en prison qui tu voudrais... Voilà ce que s'imaginent ces péquenots chez lesquels nous t'envoyons (28).

Quoique Medza se demande si les opinions du patriarche sur les bushmen de Kala n'étaient pas quelque peu exagérées, «quelque chose s'éveillait en lui comme une vocation: l'amour de l'aventure. Mais une aventure tout de même assez facile, parmi les populations naïves - ce qui est le souhait et même le vœu de tous les aventuriers " (29). Si Medza accepte la mission, ce n'est pas en tant qu'élève de l'école coloniale, ni non plus en tant qu'adepte des traditions africaines. Le recalé au baccalauréat développe à présent un ethos d'aventurier à l'image d'un "condottiere $»^{12}$, ou d'un "carbonaro $»^{13}$ ou mieux encore, d'un conquistador: "Ah! Les conquistadores! Je m'arrêtai définitivement sur cette belle race qui s'offrait à m'adopter. Quelle promotion !... » (29). Mais le choix de se mettre dans la peau d'un conquistador n'est pas gratuit. Cette posture littéraire va de pair avec celle du bon sauvage, figure mythique qui est née justement de la rencontre de la population amérindienne et des aventuriers espagnols, partis à la conquête de l'Amérique au $16^{\mathrm{e}}$ siècle ${ }^{14}$. À califourchon sur son vélo, Medza contemple l'immense panorama ouvert à ses futurs exploits de conquistador (33), tel un Pizarro, ou en l'occurrence, un "Medzarro ", qui se fraie un passage dans le pays si désiré de l'eldorado. Endosser ainsi d'abord le costume de lycéen puis le costume de conquistador revient à habiller les habitants de Kala de celui du bon sauvage dont Medza se défait du même coup. Dès son arrivée à Kala, le narrateur voit s'accomplir la prédiction du patriarche :

Dès l'abord, il me fut donné de vérifier à quel point le terme péquenot, utilisé le matin par Bikokolo, le patriarche, s'appliquait aux gens de ce pays. Juste à l'entrée du village se déroulait un spectacle saisissant non tant pas son décor que par la rude sauvagerie qui en agitait tous les acteurs (37).

Il reproduit les poncifs romantiques de l'Africain-bon sauvage qui vit au plus près de la nature au moyen de notations physiques ( «la forêt humide»), ou, en ligne indépendante, climatiques ( «il faisait très chaud ») (37) ; ou encore par les métaphores faunesques avec lesquelles il décrit les habitants en train de pratiquer, « le torse et les jambes nus", un sport inconnu évoqué par des expressions comme : "cette espèce de baobab humain qui lançait la boule..." (39), ou encore comme «la boule lancée par cette force d'orang-outang " (39), ou « tous les jeunes gens prirent le large à l'approche du rhinocéros vert, sauf Zambo qui, gambadant sur place, comme une antilope géante » (40).

38 C'est à partir de cette scénographie de l'Afrique-nature que vont se dérouler les interactions que le protagoniste va entretenir avec les autres personnages. Mais comment va-t-il se présenter à cette population naïve de Kala ? Medza est terrifié par ce spectacle de la nature et de la sauvagerie. Notre «conquistador d'occasion se convainquit tout à coup qu'il ferait mieux de redevenir le recalé au baccalauréat, d'aller se rhabiller et de remiser son armure au magasin des accessoires» (41). Pour son 
malheur, l'image que l'on projette sur lui n'est pas celui d'un loser dans laquelle il se reconnait le mieux (« il ferait mieux de redevenir le recalé au baccalauréat »). Medza se trouve de nouveau, et malgré lui, dans les habits de diplômé et soulève en tant que tel l'admiration des villageois qui l'entourent. Tout au long de son séjour à Kala, le narrateur attribue à ses hôtes de la forêt l'image du bon sauvage. Elle est dessinée à travers les thématiques stéréotypées de la nudité et de la débauche, de la paresse et de la danse, du paysage naturel et de l'esprit bon vivant, de la naïveté d'un côté, et de la sagesse, voire de la réflexion philosophique de l'autre. Mais ces représentations reviennent au narrateur par une image spéculaire qui l'inverse: dans le regard « sauvage » des habitants de la forêt, Medza est tout ce qu'ils ne sont pas.

L'ethos du narrateur se construit ainsi autour de son statut d'«étranger». Si le narrateur rapporte les interactions qu'il a entretenues avec ses hôtes des années plus tôt, son image est construite par ce qu'ils disent à son égard dans les interactions. Les habitants de Kala le voient non seulement comme un diplômé, mais aussi comme un « rupin», un « gars de la ville» (42), un « blanc» (86), voire un « colon» (105-6). Cette identité d'étranger que l'on attribue au protagoniste est précisée non pas par ce qui est explicitement dit à son égard mais par ce qui se montre à travers le dit. Le narrateur insiste, dans cette perspective, sur son altérité culturelle quand, par exemple, il raconte comment il a pris Edima pour épouse sans en être conscient, et cela en raison de son ignorance des codes de la cérémonie traditionnelle de mariage (185-89) ; ou quand il raconte comment, par pudeur, il se baignait dans la rivière avec un slip, alors que ses amis de Kala "étaient complètement nus » (61). Le narrateur se démarque du groupe par la honte qu'il éprouve devant sa nudité. Derrière son embarras d'adolescent et la situation comique qui en résulte, l'action de se couvrir prend un sens symbolique : elle positionne le narrateur à l'écart des autres qui restent dans le même état d'innocence édénique d'avant la chute. Ce dernier exemple montre comment l'image du bon sauvage sert à faire valoir celle du narrateur.

Celui-ci prend d'autres postures identitaires qui lui permettent de discourir de l'extérieur et en toute légitimité sur l'état du monde en général et sur le monde du village en particulier. Un parterre d'auditeurs demande à Medza de parler de ce qu'il a appris à l'école des Blancs.

«Pour nous», lui dit-on, «[qui] n'avons pas été à l'école, le Blanc, c'est toi fils, parce que toi seul peux nous expliquer tout ce que nous ne comprenons pas. Fais-le par amour pour nous, fils. Si tu refusais, nous aurions probablement manqué la seule chance que nous ayons jamais eue de pouvoir comprendre » (86).

Par le discours que le narrateur met dans la bouche de ses interlocuteurs ${ }^{15}$, se construit un ethos de professeur en mission civilisatrice pour sortir le sauvage de son ignorance. Cet ethos lui permet d'élaborer un discours de géographe sur les villes américaines ainsi que sur les kolkhozes russes : «Et me voilà parti sur les tracteurs, les sovkhozes, les communautés rurales, leur gestion, les résultats magnifiques [...] Mon auditoire haletait. A un moment, je pris une pause pour souffler, tant je m'étais épuisé en parlant : enseigner n'est décidément pas une sinécure » (88).

De la même manière, le narrateur développe par son discours un ethos de sociologueanthropologue qui étudie les institutions et les mœurs de cette société africaine détachée de la civilisation. On rappelle que l'ethos gère la relation entre ce que dit le sujet parlant et le fait même qu'il puisse le dire. Le discours dégage une certaine image de son énonciateur qui, inversement, donne de la crédibilité au discours qu'il énonce. 
Dans le cas qui nous concerne, c'est un ethos de sociologue-anthropologue que le narrateur construit et qui, en retour, lui confère le droit de faire des observations, par, exemple, sur la place de l'individu dans la société : « Ici, l'individu obéissait au groupe avec une complaisance qui n'eût pas manqué de surprendre Lévy-Bruhl lui-même »; (101) ou sur l'attitude contestataire de la nouvelle génération devant la chefferie "traditionnelle», souvent appuyée par le régime colonial : "Opposition anarchique, certes, verbeuse, mais qui n'en portait pas moins de fruits... » (159-160). Mais ce n'est pas tout. Le narrateur fait aussi référence à la Weltliteratur, de l'Iliade et l'Odyssée d'Homère jusqu'à Babbit (1922) de Lewis Sinclair (prix de Nobel de littérature 1930), en passant par le roman picaresque du $18^{e}$ siècle, ainsi qu'à bien d'autres genres de discours et domaines disciplinaires qui démontrent une vaste culture générale propre à un lettré plutôt qu'à un paysan de la brousse africaine.

43 A l'issue de ses aventures à Kala, le héros ne retrouve pas son village natal pour y vivre heureux le reste de ses jours. Il n'y réside que très peu avant de rompre définitivement avec sa famille et son épouse " pour recommencer une autre vie. Une vie d'errance sans fin. Errance à travers les êtres, les idées, les pays et les choses» (219). Cet ethos d'étranger, que le narrateur a construit tout au long de son récit, lui donne à la fin du roman le droit d'adopter une posture de moraliste et d'avancer son argument conclusif selon lequel le voyage était pour lui l'occasion de

découvrir au contact des péquenots de Kala, ces sortes de caricatures de l'Africain colonisé, que le drame dont souffre notre peuple, c'est celui d'un homme laissé à lui-même dans un monde qui ne lui appartient pas, un monde qu'il n'a pas fait, un monde où il ne comprend rien. C'est le drame d'unhomme laissé à lui-même sans direction intellectuelle, d'un homme marchant à l'aveuglette, la nuit, dans un quelconque New York hostile. Qui lui apprendra à ne traverser la cinquième Avenue qu'aux passages cloutés ? qui lui apprendra à déchiffrer le « Piéton, attendez » ? qui lui apprendra à lire une carte de métro, à prendre les correspondances ? (219-220)

L'image stéréotypée du bon sauvage s'appuie d'une part sur un défaut d'aptitude de l'habitant de l'Afrique rurale à la modernité, et renforce, de l'autre, l'ethos d'un narrateur africain étranger à la culture qu'il décrit. La personnalité qui se révèle à travers son discours, par contraste, ressemble beaucoup plus à celle d'un " homme du monde " qu'à celle d'un loser nomade que le narrateur prétend parfois être. Il est pourtant intéressant de noter que l'image de l'«Africain colonisé » prend un tour caricatural de l'aveu même du narrateur (" ces sortes de caricatures...») quand il évoque les " péquenots de Kala ». Il développe par là un discours critique sur sa propre tendance à attribuer aux Africains colonisés une image déformée par une simplification excessive, calquée sur le stéréotype familier du «bon sauvage ». L' " homme du monde » ne se transforme pas en «homme de nulle part ». En fin de compte, le malaise que le narrateur exprime devant le sort des Africains de la brousse procède d'un ethos d'intellectuel qui prend finalement conscience de son appartenance à la société et au monde de son temps, renonce à sa position d'étranger, et met sa pensée au service de la cause africaine.

\section{Vers un nouvel ethos d'auteur africain?}

Il reste enfin à examiner comment l'image du narrateur s'articule avec l'ethos d'auteur de Mongo Beti et si celui-ci a quelque incidence sur son ethos préalable d'« écrivain africain » tel qu'il se construit dans l'article Afrique, noire, littérature rose. L'ethos que le 
narrateur développe dans chacune des interactions présente deux faces : d'un côté celle d'un être radicalement inadapté à la vie scolaire, sociale et familiale ; de l'autre, celle d'un aventurier, d'un sociologue-anthropologue en terre inconnue, d'un éducateur progressiste en mission civilisatrice auprès des sauvages et, pour finir, d'un homme de lettres. Le fil rouge qui traverse toutes les identités qu'adopte le narrateur réside dans son statut d'étranger aux mœurs de la société africaine dans laquelle il œuvre et agit. Cette impression d'altérité culturelle relève de la manière dont le narrateur perçoit l'espace dans lequel il se situe, perception qui s'exprime entre les lignes de son discours. Son identité se développe ainsi sous l'apparence d'un inadapté à la société, qui n'est jamais à sa place là où il est, et qui en change perpétuellement - bref, par son refus de l'appartenance. L'ethos du narrateur suggère un rapport dialectique entre l'Africain et «sa » culture. Il problématise une nouvelle identité africaine présentée sous les traits d'un étranger parmi les siens, qui veut s'ouvrir à différents types de rapports entre l'homme et la culture à laquelle il est supposé appartenir, qui navigue entre les sphères culturelles.

Si le texte projette une image singulière du narrateur, une figure auctoriale se dessine aussi à travers le dispositif textuel. Elle implique un changement de point de vue et de niveau d'interaction, du discursif à l'extra-discursif. L'auteur, qui configure le texte, multiplie et justifie les images de l'Africain. La dimension argumentative de son discours relève justement de cette manière de légitimer différents ethè qui ne procèdent pas de la « vision africaine » du monde à laquelle tenaient les écrivains de la Négritude. Qui plus est, l'image caricaturale qu'il donne de l'Africain de la brousse, à travers le discours qu'il met dans la bouche du narrateur, déconstruit, par l'effet parodique, le discours stéréotypé de l'« écrivain africain ». Ainsi, le discours littéraire n'est plus perçu dans la perspective tracée par les partisans de la Négritude comme moyen d'exalter la Culture par son talent artistique, il permet désormais à l'auteur de se repositionner à l'écart de la tribu des "écrivains africains » dans une recherche permanente de nouveaux modes d'expression. L'image littéraire de l'Afrique et de l'Africain est ainsi perpétuellement remise en cause dans le travail discursif d'un écrivain qui, au tournant des Indépendances, veut renégocier sa place dans le champ littéraire français.

\section{BIBLIOGRAPHIE}

Amossy, Ruth. 2010. La Présentation de soi. Ethos et identité verbale (Paris : PUF)

Amossy, Ruth. 2009. «La double nature de l'image d'auteur », Argumentation et Analyse du Discours 3 [en ligne : http://aad.revues.org/656]

Amossy, Ruth (éd.). 2002. Pragmatique et analyse des textes (Tel-Aviv : Presses de l'Université de TelAviv)

Amossy, Ruth \& Dominique Maingueneau (éds.). 2004. L'analyse du discours dans les études littéraires(Toulouse : Presses Universitaires du Mirail) 
Amossy, Ruth \& Dominique Maingueneau, 2009. « Autour des 'scénographies auctoriales' : entretien avec José-Luis Diaz, auteur de L'Écrivain imaginaire (2007) », Argumentation et Analyse du Discours 3 [en ligne : http://aad.revues.org/656]

Amossy, Ruth (éd.). 1999. Images de soi dans le discours : la construction de l'ethos (Paris-Lausanne : Delachaux \& Niestlé)

Beti, Mongo. 1957 [1985]. Mission terminée (Paris : Corrêa, Buchet-Chastel)

Beti, Mongo. 1964. Mission to Kala (London, Ibadan, Nairobi : Heinemann Educational Books)

Biyidi, Alexandre. 1955. « Afrique noire, littérature rose ». Présence Africaine 1-2, 133-145

Brière, Éloïse. 1979. «La réception critique de l'œuvre de Mongo Béti », Euvres et critique. Réception critique de la littérature africaine et antillaise d'expression française 3-4, 79-88

Chauraudeau, Patrick \& Dominique Mangueneau. 2002. Dictionnaire d'analyse du discours (Paris : Seuil)

Césaire, Aimé. 1935. « Nègreries. Jeunesse noire et assimilation », L'Étudiant noir. Journal de l'Association des Étudiants Martiniquais en France 1

Damas, Contran Léon. 1937. Pigments (Paris : Lévi Mano)

Diaz, José-Luis. 2000. « Le poète comme roman », Lavialle, Nathalie \& Jean-Benoît Puech (éds.).

L'auteur comme cuvre : l'auteur, ses masques, son personnage, sa légende (Orléans : Presses

Universitaires d'Orléans), 55-68

Diaz, José-Luis). 2007. L'Écrivain imaginaire : scénographies auctoriales à l'époque romantique (Paris : Champion)

Diaz, José-Luis, Declercq, Elien \& Karen Vandemeulebroucke. 2012. « De l'écrivain au traducteur imaginaires. Entretien avec José-Luis Diaz au sujet de sa théorie de l'auteur », Interférences littéraires/Literaire interferenties 9 [en ligne : http://www.interferenceslitteraires.be/node/179]

Dhondt, Reindert \& Beatrijs Vanacker. 2013. «Ethos : pour une mise au point conceptuelle et méthodologique », Contextes 13 [en ligne : http://contextes.revues.org/5674]

Dhondt, Reindert, Katrien Horemans, Beatrijs Vanacker \& Karen Vandemeulebroucke. 2013.

«Avant-propos », Contextes 13 [en ligne : http://contextes.revues.org/5674]

Genette, Gérard. 1987. Seuils (Paris : Editions du Seuil)

Kane, Mouhamadou. 1974. « Les Formes traditionnelles du roman africain », Revue de Littérature comparée 3-4, 536-568

Kahan, Bokobza Michele. 2009. «Introduction », Argumentation et Analyse du Discours 3 [en ligne : http://aad.revues.org/656]

Kesteloot, Lilian. 1975 [1963]. Les Écrivains noirs de langue française : naissance d'une littérature (Bruxelles : Éditions de l'Université de Bruxelles)

Killam Douglas, Gordon. 1973. African Writers on African Writing (London, Ibadan, Nairobi :

Heinemann Educational Books)

Le Bris, Michel \& Jean Rouaud. 2007. Pour une littérature-monde (Paris : Gallimard)

Lejeune, Philippe. 1980. Je est un autre. L'Autobiographie de la littérature aux médias (Paris : Seuil)

Maingueneau, Dominique. 2004. Le discours littéraire. Paratopie et scène d'énonciation (Paris : Armand Colin) 
Maingueneau, Dominique. 1993. Le contexte de l'œuvre littéraire. Énonciation, écrivain, société (Paris : Dunod)

Maingueneau, Dominique. 2002. « Problèmes d'ethos » Pratiques 113-114, 55-67

Maingueneau, Dominique, Reindert Dhondt \& David Martens. 2012. « Un réseau de concepts. Entretien avec Dominique Maingueneau au sujet de l'analyse du discours littéraire », Interférences littéraires / Literaire interferenties 8 [en ligne : http://interferenceslitteraires.be/node/162]

Meizoz, Jérôme). 2007. Postures littéraires. Mises en scène modernes de l'auteur (Genève : Slatkine, 2007)

Meizoz, Jérôme. 2011. La fabrique des singularités. Postures littéraires II (Genève : Slatkin)

Meizoz, Jérôme \& David Martens. 2011b. « La fabrique d'une notion. Entretien avec Jérôme Meizoz au sujet du concept de 'posture' ", Interférences littéraires / Literaire interferenties, 6 [en ligne : http://www.interferenceslitteraires.be/node/19]

Michaud, Guy (éd.). 1978. Négritude : traditions et développement (Paris : PUF)

Mongo-Mboussa, Boniface. 2002. Désir d'Afrique (Paris : Gallimard, coll. Continents noirs)

Mouralis, Bernard. 1981. Littérature et développement : essai sur le statut, la fonction et la représentation de la littérature négro-africaine d'expression française (Lille : Atelier reproduction des thèses Université de Lille III)

Mouralis, Bernard. 1981. Comprendre l'œuvre de Mongo Beti (Paris : Saint-Paul)

Riesz, János \& Ulla Schild (éds). 1996. Genres autobiographiques en Afrique (Berlin : Reimer Verlag)

Sallah Hassan D. 1999. « The Cultural Politics of the Early Présence Africaine, 1947-55 », Research in African Literatures 30, 194-221

Schüler, Thorsten. 2012. « "La littérature africaine n'existe pas”, ou l'effacement des traces identitaires dans les littératures africaines subsahariennes de langue française ", Études littéraires africaines 32 (Metz : Université de Lorraine)

Van Gorp, Hendrik, Dirk Delabastita, Lieven D’hulst, Rita Ghesquiere, Rainier Grutman \& Georges Legros. 2001. Dictionnaire des termes littéraires (Paris : Honoré Champion)

\section{NOTES}

1. Voir notamment l'article de Reindert Dhondt et Beatrijs Vanacker, 2013. «Ethos : pour une mise au point conceptuelle et méthodologique "

2. Voir le site de la maison d'éditions: http://www.gallimard.fr/catalog/html/actu/index/ index_continentsnoirs.html

3. C'est aussi le titre de l'interview telle qu'elle a été publiée dans l'ouvrage de Boniface MongoMboussa Désir d'Afrique (2002).

4. L'auteur cite les noms de Léopold Senghor, Camara Laye et Bernard Dadié.

5. Signalons que l'article Afrique noire, littérature rose est suivi du roman Le pauvre Christ de Bomba (1956). Il importe d'observer par ailleurs l'incidence de cette œuvre romanesque dans la formation (ou transformation) de l'ethos préalablede Mongo Beti, l'auteur de Mission terminée. Cette étude importante qui reste à faire dépasse les limites de cet article

6. «Tout ce par quoi un texte se fait livre et se propose comme tel à ses lecteurs » (Genette 1987 : 7)

7. Je m'appuie ici sur le Dictionnaire des termes littéraires (2001) 
8. Le commentaire éditorial de la quatrième de couverture de 1985 est une version réduite de celle de 1957. Elle supprime les détails mentionnés dans la première édition sur l'accueil que les habitants de Kala réservent au héros, en particulier, sur la relation qu'il entretient avec son oncle et, plus piquant encore, avec les femmes du village. Ces détails sont réintégrés à l'intérieur du livre et suivent la notice biographique que l'éditeur introduit dans la toute première page du livre.

9. Dans le commentaire éditorial de 1985, Mongo Beti est un écrivain noir couronné de succès : « Mission terminée de Mongo Beti, un des meilleurs écrivains noirs contemporains...» Cette manière de classer les écrivains africains dans un ensemble est évidente dans le monde anglophone. Le roman Mission terminée est ainsi publié dans sa version anglaise sous le titre Mission to Kala dans la collection African Writers Series initiée par les Éditions Heinmann Educational Books LTD.

10. A l'exemple de Continents noirs chez Gallimard, dans le monde anglophone on classe également les écrivains noirs dans un ensemble regroupé. C'est le cas de la collection African Writers Series.

11. Toutes les citations se rapportent à la présente édition de Mission terminée

12. Au Moyen Âge, Chef de soldats mercenaires, en Italie.

13. Membre d'une société secrète italienne qui, au début du $\mathrm{XIX}^{\mathrm{e}}$ siècle, combattait pour la liberté nationale.

14. Voir à ce sujet l'ouvrage de Bernard Mouralis, Montaigne et le mythe du bon sauvage. De l'Antiquité à Rousseau, Paris : Bordas, 1999.

15. Patrick Charaudeau relève l'ambiguïté de la notion d'interlocuteur (2002). Pour ce qui concerne notre recherche, la notion d'interlocuteurs, au pluriel, se réfère aux acteurs d'un acte de communication, et qui participent à l'interaction

\section{RÉSUMÉS}

$\mathrm{Au}$ tournant des Indépendances (1950-1960), l'ethos que l'auteur africain construit dans son discours littéraire interagit avec une image préexistante, développée précédemment par les écrivains de la Négritude. À partir d'une analyse de Mission terminée de Mongo Beti, cet article se propose d'éclairer les interrelations, voire les tensions, entre l'ethos préalable (de type collectif) de l'« écrivain africain » et celui que projette, dans son discours, un auteur qui veut s'en écarter. Notre attention se concentrera sur les conditions d'émergence d'une œuvre dont l'auteur cherche à se repositionner, d'abord, dans le champ littéraire puis, plus généralement, dans l'espace social, s'efforçant de refaçonner sa double identité d'écrivain et d'Africain. L'article se propose ainsi d'éclairer non seulement le rapport qui se noue entre un texte et son lecteur mais aussi certaines dimensions institutionnelles de la « littérature africaine ».

At the time of Independence (1950-1960), the ethos that the African author constructs throughout his literary discourse interacts with a preexisting image previously developed by the writers of the Negritude. Studying the case of Mission to Kala by Mongo Beti, this article examines the interrelations and the tensions between the prior ethos of the "African writer" and the one that Mongo Beti projects in this specific novel. The analysis focuses on the conditions that allow the writing of a novel whose author is looking to reposition himself in the literary French field and, in a broader sense, in the public sphere. By doing so, he reconstructs his identity as a writer as 
well as an African. Finally, by considering the relationship between the text and its reader we wish to shed light on some institutional dimensions of the so-called "African literature".

INDEX

Keywords : African literature, author, ethos, négritude, stereotype, writer

Mots-clés : auteur, écrivain, ethos, littérature africaine, négritude, stéréotype

\section{AUTEUR}

TAL SELA

ADARR, Université de Tel-Aviv 